\title{
ORIGINAL ARTICLE Wnt5a induces ROR1 to complex with HS1 to enhance migration of chronic lymphocytic leukemia cells
}

\author{
MK Hasan ${ }^{1}$, J Yu ${ }^{1}$, L Chen ${ }^{1}$, Bing Cui ${ }^{1}$, GF Widhopf $I^{1}$, L Rassenti ${ }^{1}$, Z Shen ${ }^{2}$, SP Briggs ${ }^{2}$ and TJ Kipps ${ }^{1}$
}

ROR1 (receptor tyrosine kinase-like orphan receptor 1) is a conserved, oncoembryonic surface antigen expressed in chronic lymphocytic leukemia (CLL). We found that ROR1 associates with hematopoietic-lineage-cell-specific protein 1 (HS1) in freshly isolated CLL cells or in CLL cells cultured with exogenous Wnt5a. Wnt5a also induced HS1 tyrosine phosphorylation, recruitment of ARHGEF1, activation of RhoA and enhanced chemokine-directed migration; such effects could be inhibited by cirmtuzumab, a humanized anti-ROR1 mAb. We generated truncated forms of ROR1 and found its extracellular cysteine-rich domain or kringle domain was necessary for Wnt5a-induced HS1 phosphorylation. Moreover, the cytoplamic, and more specifically the proline-rich domain (PRD), of ROR1 was required for it to associate with HS1 and allow for F-actin polymerization in response to Wnt5a. Accordingly, we introduced single amino acid substitutions of proline (P) to alanine (A) in the ROR1 PRD at positions 784, 808, 826, 841 or 850 in potential SH3-binding motifs. In contrast to wild-type ROR1, or other ROR1 ${ }^{\mathrm{P} \rightarrow \mathrm{A}}$ mutants, ROR1 ${ }^{\mathrm{P}(841) \mathrm{A}}$ had impaired capacity to recruit HS1 and ARHGEF1 to ROR1 in response to Wnt5a. Moreover, Wnt5a could not induce cells expressing ROR1 $1^{\mathrm{P}(841) \mathrm{A}}$ to phosphorylate HS1 or activate ARHGEF1, and was unable to enhance CLL-cell motility. Collectively, these studies indicate HS1 plays an important role in ROR1-dependent Wnt5a-enhanced chemokine-directed leukemia-cell migration.

Leukemia (2017) 31, 2615-2622; doi:10.1038/leu.2017.133

\section{INTRODUCTION}

ROR1 (receptor tyrosine kinase-like orphan receptor 1) is an evolutionarily conserved, type-I membrane protein that is expressed during embryogenesis, where it plays a key role in skeletal and neural organogenesis. $^{1-4}$ Expression of ROR1 attenuates during fetal development and, with few exceptions, ${ }^{5}$ is negligible on most normal postpartum tissues. ${ }^{6}$ However, we and others have found the leukemia cells of most patients with chronic lymphocytic leukemia (CLL) express ROR $1{ }^{6-8}$ suggesting it may play a role in pathogenesis. Consistent with this notion are studies showing that expression of ROR1 can enhance disease progression in mouse models of this leukemia, ${ }^{9}$ and in patients with CLL. ${ }^{10}$

We found that ROR1 can serve as a receptor for Wnt5a, ${ }^{6}$ which prior studies showed could induce non-canonical Wnt signaling involved in directional cell migration and planar-cell polarity. ${ }^{11}$ More recent studies on CLL cells found Wnt5a could induce ROR1 to form hetero-oligomers with ROR2 and recruit and activate guanine exchange factors (GEFs), resulting in activation of Rho GTPases and enhanced leukemia-cell migration and proliferation. ${ }^{12}$ These effects of Wnt5a on CLL cells could be inhibited by cirmtuzumab, a humanized mAb specific for ROR1 that specifically could block the capacity of Wnt5a to enhance leukemia-cell proliferation or migration. However, the cytoplasmic proteins enabling Wnt5a to enhance ROR1-dependent leukemiacell migration were unknown.

Important for the organization of the cytoskeleton required for migration and possibly planar-cell polarity is hematopoieticlineage-cell-specific protein 1 (HS1). HS1 is a cytoplasmic protein that can be undergo tyrosine phosphorylation and promote polymerization and rearrangement of the actin cytoskeleton required for cell migration. ${ }^{13-17} \mathrm{HS} 1$ also contains an SH3 domain, which allows it to bind characteristic motifs (-P-X-X-P-), which often are found in the proline-rich domains (PRDs) of other proteins. ${ }^{18-20} \mathrm{HS} 1$ also is expressed in CLL cells. ${ }^{21-24}$ Moreover, expression and phosphorylation of HS1 correlates with enhanced CLL-cell migration and unfavorable prognosis for patients with CLL. ${ }^{22,23,25-27}$ Here we report the finding that Wnt5a induces ROR1 to associate with HS1, which undergoes tyrosine phosphorylation and recruits/activates ARHGEF1 to promote F-actin polymerization and leukemia-cell migration.

\section{MATERIALS AND METHODS}

\section{Immunoprecipitation analysis}

Immunoprecipitation analysis was performed as described. ${ }^{9}$ Cells were lysed in a buffer containing $1 \%$ Nonidet P-40, $10 \mathrm{~mm}$ Tris-HCl (pH 7.5), $50 \mathrm{~mm} \mathrm{NaCl}$ and $1 \mathrm{~mm}$ EDTA with protease inhibitors (Roche, Applied Science, Mannheim, Germany). The lysates were cleared by centrifugation at $16000 \mathrm{~g}$ for $15 \mathrm{~min}$. Immune precipitates were isolated using protein A agarose beads, followed by immunoblot or mass spectrometry analysis. Antibodies for immune precipitation were as follows: the anti-ROR1 antibodies (cirmtuzumab or 4A5) were generated in our laboratory; the anti-HS1 or ARHGEF1 antibody was obtained from Cell Signaling Technology (Danvers, MA, USA).

\section{Immunoblot analysis}

Western blot analysis was performed as described. ${ }^{9}$ Equal amounts of total protein from each sample were separated by SDS-PAGE and blotted onto polyvinylidene difluoride membrane. Western blot analysis was performed using primary mAbs specific for ROR1 (Cell Signaling), HS1 (Cell Signaling), phospho HS1 (Y378) (OriGene, Rockville, MD, USA), ARHGEF1 (Cell Signaling) or $\beta$-actin (Cell Signaling), which were detected using secondary antibodies conjugated with horseradish peroxidase (Cell Signaling Technology).

\footnotetext{
${ }^{1}$ Moores Cancer Center, University of California San Diego, La Jolla, CA, USA and ${ }^{2}$ Section of Cell and Developmental Biology, University of California San Diego, La Jolla, CA, USA. Correspondence: Dr TJ Kipps, Medicine, University of California San Diego, 3855 Health Sciences Drive \#0820, La Jolla, CA, USA. 
a

\begin{tabular}{rcc} 
& \multicolumn{2}{l}{ I.P. antibody } \\
\cline { 2 - 3 } Ctrl-IgG & + & - \\
Anti-ROR1 & - & +
\end{tabular}
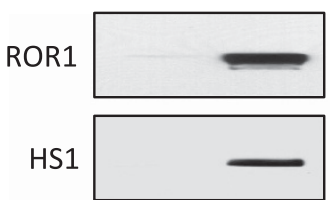

d

C

\section{I.P: Anti-ROR1 antibody}

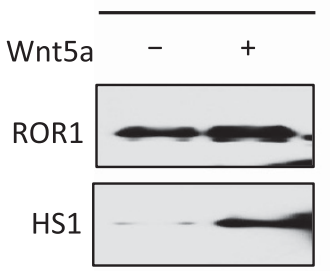

b

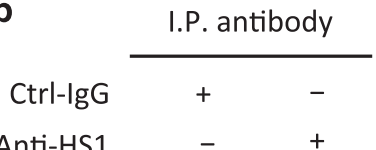

Anti-HS1

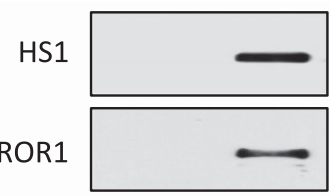

I.P. antibody

$\begin{array}{ccccc}\text { Ctrl-IgG } & - & - & - & + \\ \text { Anti-ROR1 } & + & + & + & -\end{array}$

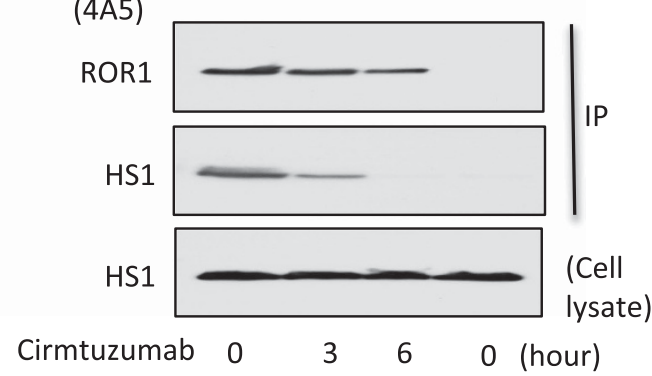

e

DAPI

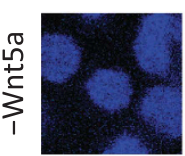

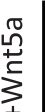

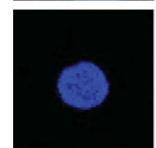

$\stackrel{\text { in }}{\stackrel{1}{*}}$
ROR1

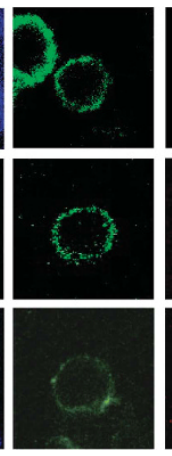

HS1

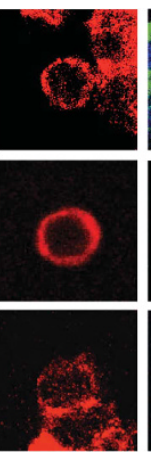

Merge

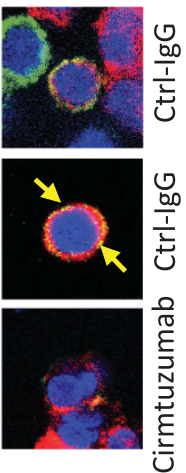

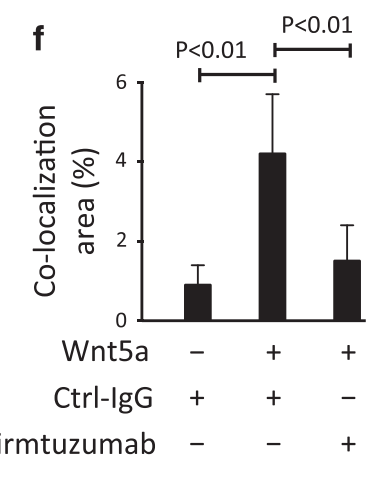

Figure 1. Association of ROR1 with HS1 in primary CLL cells. (a) Immunoblot analysis of anti-ROR1 ip or control lgG (Ctrl-lgG) ip, as indicated at the top, using lysates prepared from freshly isolated primary CLL cells; the membranes were probed with anti-ROR1 or anti-HS1 antibody, as indicated on the left. (b) Immunoblot analysis of anti-HS1 ip or Ctrl-lgG ip, as indicated at the top, using lysates prepared from freshly isolated primary CLL cells; the membranes were probed with anti-ROR1 or anti-HS1 antibody, as indicated on the left. (c) Immunoblot analysis of anti-ROR1 ip using lysates prepared from overnight, serum-starved primary CLL cells that subsequently were treated for 30 min without (-) or with (+) Wnt5a $(100 \mathrm{ng} / \mathrm{ml})$, as indicated on the top; the membranes were probed with anti-ROR1 or anti-HS1 antibody, as indicated on the left. (d) Immunoblot analysis of anti-ROR1 (4A5) ip or Ctrl-IgG ip, as indicated at the top, using lysates prepared from freshly isolated primary CLL cells that had been treated with cirmtuzumab for the times indicated at the bottom (in hours); membranes were probed with anti-ROR1 or anti-HS1 antibody, as indicated on the left. An immunoblot of the whole-cell lysates of the CLL treated with cirmtuzumab and probed with anti-HS1 mAb is provided in the bottom panel. (e) Confocal microscopy of serum-starved CLL cells stained with fluorochrome-conjugated mAb specific for ROR1 (green) or HS1 (red) after the cells were treated with Ctrl-lgG or cirmtuzumab $(10 \mu \mathrm{g} / \mathrm{ml})$, as indicated on the right, without (-) or with (+) Wnt5a $(100 \mathrm{ng} / \mathrm{ml})$, as indicated on the left; co-localized staining for ROR1 and HS1 in the same pixel is depicted in yellow (arrow). Objective, $\times 100$. Scale bar: $2 \mu \mathrm{m}$. (f) The histograms depict the proportions of the area that has co-localized staining for ROR1 and HS1 in primary CLL cells, as indicated on the left. Data are shown as the mean \pm s.d. of CLL cells from each of three patients after treatment with Ctrl-lgG or cirmtuzumab $(10 \mu \mathrm{g} / \mathrm{ml})$ without $(-)$ or with $(+)$ Wnt5a $(100 \mathrm{ng} / \mathrm{ml}) ; P<0.01$, as calculated using the two-tailed Student's $t$-test.

\section{Cell migration assay}

The cell migration assay was preformed as described. ${ }^{12,28}$ Briefly, a total of $5 \times 10^{5}$ cells were washed twice, cultured overnight in serum-free medium and then treated with or without Wnt5a $(200 \mathrm{ng} / \mathrm{ml})$ for $30 \mathrm{~min}$. The cells then were placed into the top chamber of a Transwell culture polycarbonate insert with $6.5-\mathrm{mm}$ diameter and $5 \mu \mathrm{m}$ pore size (Corning, Inc., Corning, NY, USA). Cells were incubated for $2 \mathrm{~h}$ in serum-free medium at $37^{\circ} \mathrm{C}$ and $5 \% \mathrm{CO}_{2}$, and the migration toward chemokine (CXCL12,
$200 \mathrm{ng} / \mathrm{ml}$ or CCL21, $200 \mathrm{ng} / \mathrm{ml}$ ) was analyzed by flow cytometry. The percentage of migrating cells was calculated as the number of migrated cells in response to chemokine divided by the total number of input cells.

\section{Study approval}

Blood samples were collected from CLL patients at the Moores Cancer Center who satisfied diagnostic and immuno-phenotypic criteria for 
a

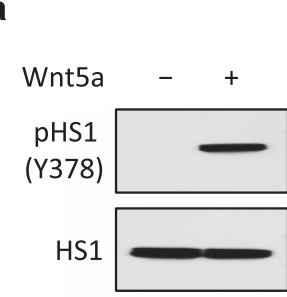

$\begin{array}{rrrr}\text { b Wnt5a } & - & + & + \\ \text { Cirmtuzumab } & - & - & + \\ \text { Ctrl-IgG } & + & + & -\end{array}$
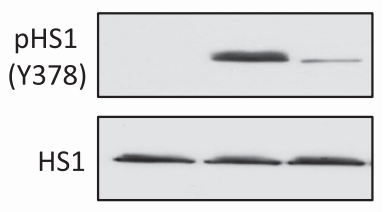

C

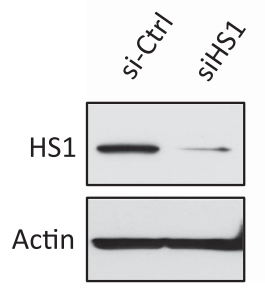

Figure 2. Wnt5a induces ROR1-dependent phosphorylation of HS1 and enhances chemokine-directed leukemia-cell migration. (a) Immunoblot analysis of lysates prepared from overnight, serum-starved primary CLL cells that subsequently were treated for $5 \mathrm{~min}$ without $(-)$ or with $(+)$ Wnt5a $(100 \mathrm{ng} / \mathrm{ml})$, as indicated on the top; the membranes were probed with anti-HS1 or anti-phospho HS1 (Y378) antibody, as indicated on the left. (b) Immunoblot analysis of lysates prepared from overnight, serum-starved primary CLL cells that subsequently were treated with Ctrl-lgG or cirmtuzumab $(10 \mu \mathrm{g} / \mathrm{ml})$, without $(-)$ or with $(+)$ Wnt5a $(100 \mathrm{ng} / \mathrm{ml})$, as indicated on the top; the membranes were probed with anti-HS1 or anti-phospho HS1 (Y378) antibody, as indicated on the left. (c) Immunoblot analysis of lysates prepared from CLL cells transfected 72 -h previously with control siRNA or siRNA targeting $\mathrm{HS} 1$; membranes were probed with anti-HS1 or anti- $\beta$-actin antibody, as indicated on the left. Cell viability was over $80 \%$ both in control or HS1-siRNA transfected cells. (d) CLL-cell migration in response to CXCL12 (200 ng/ml) was assessed without (-) or with (+) addition of exogenous Wnt5a $(200 \mathrm{ng} / \mathrm{ml})$, as indicated at the bottom. Data are shown as mean \pm s.d. from three independent experiments of CLL cells from each of six patients. $P<0.05 ; P<0.01$; $P<0.001$, as assessed by two-tailed Student's $t$-test.

common B-cell CLL and who provided written informed consent, in compliance with the Declaration of Helsinki and the Institutional Review Board (IRB) of the UCSD (IRB approval number 080918).

\section{RESULTS}

Wnt5a induces ROR1 to associate with HS1 in primary CLL cells We performed mass spectrometry-based proteomic analysis on anti-ROR1 immune precipitates from CLL-cell lysates and detected HS1 in addition to ROR1 (Supplementary Figure S1A). Immunoblot analysis of anti-ROR1 or anti-HS1 immune precipitates confirmed that ROR1 associated with HS1 in freshly isolated primary CLL cells (Figures 1a and b). However, when we examined anti-ROR1 immune precipitates from lysates of CLL cells cultured overnight in Wnt5a-deficient media, we found that HS1 was no longer associated with ROR1. On the other hand, anti-ROR1 immune precipitates made from lysates of serum-starved CLL cells that were treated for $30 \mathrm{~min}$ with or without exogenous Wnt5a showed that Wnt5a could induce ROR1 to associate with HS1 (Figure 1c). Treatment of the CLL cells with another non-cross-blocking anti-ROR1 mAb, cirmtuzumab, inhibited the

capacity of Wnt5a to induce ROR1 to associate with HS1 in freshly isolated CLL cells (Figure 1d). These results were corroborated using fluorescence confocal microscopy, which demonstrated that Wnt5a induced co-localization of ROR1 with HS1 in CLL cells cultured with exogenous Wnt5a, and that such co-localization could be inhibited by treatment of the CLL cells with cirmtuzumab (Figures $1 \mathrm{e}$ and $\mathrm{f}$ ).

Wnt5a induces HS1 phosphorylation and enhances ROR1dependent cell migration

Prior studies found that HS1 may be constitutively phosphorylated in CLL cells. ${ }^{25,26}$ Moreover, patients with CLL cells that had higher than average ratios of phosphorylated to non-phosphorylated HS1 appeared to have relatively aggressive clinical-disease progression. ${ }^{26}$ We confirmed that HS1 is phosphorylated at Y378 in freshly isolated CLL cells. However, culture of CLL cells in serumfree media lacking Wnt5a resulted in attrition of the levels of phosphorylation of HS1 at Y378 over time (Supplementary Figure S1B). On the other hand, treatment of these CLL cells with exogenous Wnt5a induced tyrosine phosphorylation of HS1 in such serum-starved CLL cells (Figure 2a). However, treatment of these cells with cirmtuzumab inhibited the capacity of Wnt5a to induce tyrosine phosphorylation of HS1 (Figure 2b), indicating the observed effect of Wnt5a on HS1 phosphorylation was dependent on ROR1.

Prior studies demonstrated that treatment of CLL cells with Wnt5a could enhance migration directed by chemokines, such as CXCL12, and that such effects could be inhibited by cirmtuzumab. ${ }^{12}$ We corroborated these findings (Supplementary Figure S2A), and also found that the capacity of Wnt5a to enhance chemokine-directed CLL-cell migration could be inhibited by reducing expression of HS1 with specific siRNA, but not by control siRNA (Figures 2c and d), suggesting a dependency on HS1 for Wnt5a-enhanced chemokine-directed CLL-cell migration. On the other hand, ibrutinib, a drug that can block BTK and inhibit B-cell receptor signaling (Supplementary Figure S3A), ${ }^{29}$ could not inhibit the capacity of Wnt5a to induce phosphorylation of HS1 (Y378) or enhance the migration of CLL cells in vitro (Supplementary Figures S3B-D).

Tyrosine phosphorylation of HS1 in MEC1 or MEC1-ROR1 cells MEC1 cells are derived from CLL cells and have been used as a model system for studying this leukemia. ${ }^{30}$ However, our prior studies found that MEC1 does not express ROR1 unless transfected with an expression vector encoding this orphan receptor. ${ }^{12}$ These studies also found MEC 1 cells express high levels of Wnt5a, obviating the addition of exogenous Wnt5a to the culture media. $^{12}$ Immunoblot analysis of anti-ROR1 or anti-HS1 immune precipitates revealed that ROR1 could interact with HS1 in MEC1-ROR1 cells (Figures 3a and b). As expected, ROR1 was not observed in anti-HS1 immune precipitates of lysates of MEC1 cells (Figure 3c). Of note, we found MEC1-ROR1 cells had substantially higher levels of phosphorylated HS1 than MEC1 cells, which had negligible levels of phosphorylated HS1 (Figure 3d). Consistent with this effect being induced by autocrine Wnt5a, we found that an antibody capable of neutralizing Wnt5a could induce dissociation of ROR1 with HS1 (Figure 3e) and attrition in the levels of phosphorylated HS1 over time (Figure 3f).

ROR1/HS1 interacts with ARHGEF1 and induces activation of RhoA As noted in prior studies, ${ }^{12}$ MEC1-ROR1 cells had enhanced activation of RhoA relative to that of MEC1 parental cells (Supplementary Figure S2B). These studies also demonstrated that ARHGEF1 was recruited to ROR1 following treatment with Wnt5a, where it became activated and capable of effecting activation of RhoA. ${ }^{12}$ Immunoblot analysis of anti-HS1 
a

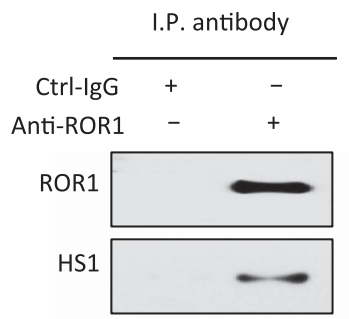

b

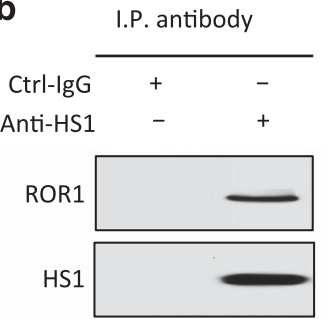

e d

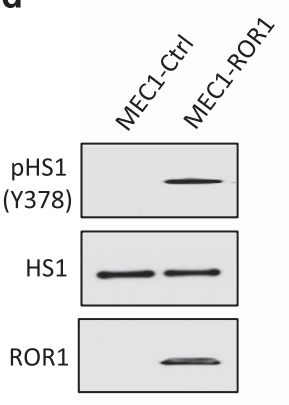

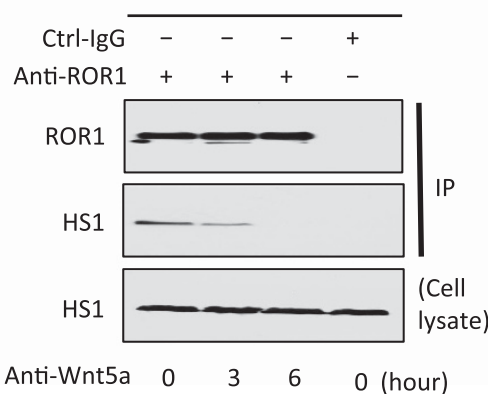

C

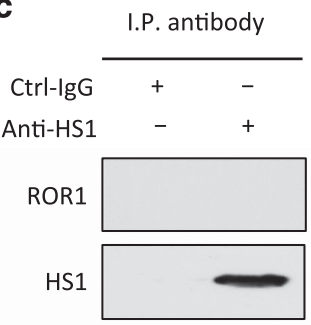

f

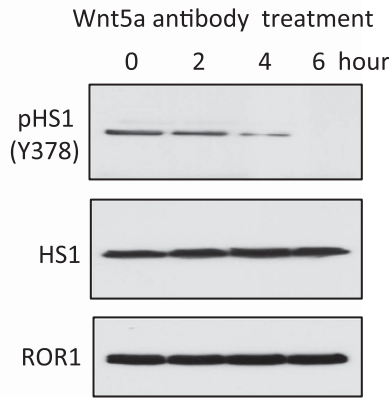

Figure 3. ROR1 in MEC1-ROR1 cells associates with HS1, which undergoes Wnt5a-dependent phosphorylation at Y378. (a) Immunoblot analysis of anti-ROR1 ip or Ctrl-lgG ip, as indicated at the top, using lysates prepared from MEC1-ROR1 cells; the membranes were probed with anti-ROR1 or anti-HS1 antibody, as indicated on the left. (b) Immunoblot analysis of anti-HS1 ip or Ctrl-IgG ip, as indicated at the top, using lysates prepared from MEC1-ROR1 cells; membranes were probed with anti-ROR1 or anti-HS1 antibody, as indicated on the left. (c) Immunoblot analysis of anti-HS1 ip or Ctrl-IgG ip, as indicated at the top, using lysates prepared from ROR1-negative cell line, MEC1; the membranes were probed with anti-ROR1 or anti-HS1 antibody, as indicated on the left. (d) Immunoblot analysis of lysates prepared from MEC1 or MEC1-ROR1 cells, as indicated on the top; membranes were probed with anti-HS1, anti-phospho HS1 (Y378), or ROR1 antibody, as indicated on the left. (e) Immunoblot analysis of anti-ROR1 ip or Ctrl-lgG ip, as indicated at the top, using lysates prepared from MEC1-ROR1 cells that had been treated with Wnt5a neutralizing antibody $(2 \mu \mathrm{g} / \mathrm{ml}, \mathrm{R} \& \mathrm{D}$, Minneapolis, MN, USA) for the times indicated at the bottom (in hours); membranes were probed with anti-ROR1 or anti-HS1 antibody, as indicated on the left. An immunoblot of the whole-cell lysates of the MEC1-ROR1 cells treated with Wnt5a neutralizing antibody and probed with anti-HS1 mAb is provided in the bottom panel. (f) Immunoblot analysis of lysates prepared from MEC1-ROR1 cells that had been treated with Wnt5a neutralizing antibody ( $2 \mu \mathrm{g} / \mathrm{ml}$, R \& D) for the times indicated at the top (in hours); membranes were probed with anti-HS1, anti-phospho HS1 (Y378), or anti-ROR1 antibody, as indicated on the left.

or anti-ARHGEF1 ip from lysates of freshly isolated CLL cells revealed that HS1 was complexed with ARHGEF1 (Figures 4a and $b$ ). We also found that reducing expression of HS1 using specific siRNA inhibited the capacity of Wnt5a to induce activation of RhoA by ip generated from anti-ARHGEF1 (Figure 4c) and inhibited activation of RhoA in MEC1-ROR1 cells (Figure 4d), indicating that HS1 contributed to Wnt5a-induced RhoA activation.

Structural domains of ROR1 required for recruitment and activation of HS1

We examined the structural domains of ROR1 that were required for it to interact with HS1. For this, we transfected MEC1 cells with a vector encoding either wild-type ROR1 or any one of various mutant forms of ROR1 (Figure 5a). Comparable levels of wild-type (W/T) or truncated forms of ROR1 were expressed by each of the various transfectants (Figure $5 \mathrm{~b}$ and Supplementary Figure S4A). Moreover, comparable levels of HS1 were found in each of the various MEC1 transfectants and in the MEC1 parental cell line (Figure $5 \mathrm{~b}$ ). We found that anti-ROR1 ip from lysates of MEC1- $\triangle$ CRD-ROR1 or MEC1- $\triangle K N G-R O R 1$, respectively, expressing a mutant form of ROR1 lacking its extracellular cystein-rich-domain (CRD) or Kringle domain (KNG), contained HS1, as did the anti-ROR1 ip from lysates of MEC1 cells transfected with W/T ROR1 (Figure 5b). However, the amount of HS1 protein found in the anti-ROR1 ip from lysates of MEC1- $\triangle$ CRD-ROR1 cells was substantially less than that noted from lysates of MEC1-ROR1 or MEC1- $\triangle K N G-R O R 1$ cells. Furthermore, anti-ROR1 ip from lysates of MEC1- $\triangle$ C-ROR1 or MEC1- $\triangle$ PRD-ROR1 cells, respectively, expressing a mutant ROR1 lacking its cytoplasmic domain (CD) or PRD, did not contain any detectable HS1. Finally, in contrast to MEC1-ROR1 cells, each of MEC1 cells expressing truncated forms of ROR1 did not have detectable levels of phosphorylated HS1, as noted for MEC1 cells lacking ROR1 (Figure $5 b$ ).

We examined MEC1 cells or MEC1-ROR1 with W/T ROR1 or any one of the various truncated mutant forms of ROR1 for F-actin polymerization following treatment with CCL21, which can induce chemotaxis of MEC1 cells or MEC1-ROR1 cells. ${ }^{12}$ Relatively low levels of F-actin polymerization were induced by CCL21 in MEC1 cells compared with that observed in MEC1-ROR1 cells (Figure 5c). Moreover, the levels of F-actin polymerization observed in MEC1 cells expressing any one of the truncated mutant forms of ROR1 were comparable to that observed in MEC1 cells lacking expression of ROR1.

Proline-841 of ROR1 is necessary for effective recruitment and activation of HS1

HS1 contains an SH3 domain, which can bind to -P-X-X-P- motifs found in many proteins with PRDs, such as ROR1. ${ }^{1,18-20,31}$ Because the PRD of ROR1 was required for ROR1 to complex with HS1, we generated various mutant forms of ROR1 with proline $(P)$ to alanine (A) substitutions at the -P-X-X-P- motifs identified in ROR1, namely at positions 784, 808, 826, 841 and 850 (Figures $6 a-C$ and Supplementary Figure S4B). ROR1 with $\mathrm{P} \rightarrow \mathrm{A}$ substitutions at 784, 808 and 826 bound to HS1 as effectively as W/T ROR1 (Supplementary Figure S5A). MEC1 cells transfected with each of these forms of ROR1 had enhanced chemotaxis in response to CCL21, as did MEC-ROR1 cells expressing W/T ROR1 
a

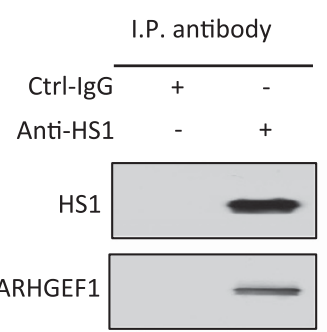

C - Buffer - siHS1 - si-Ctrl

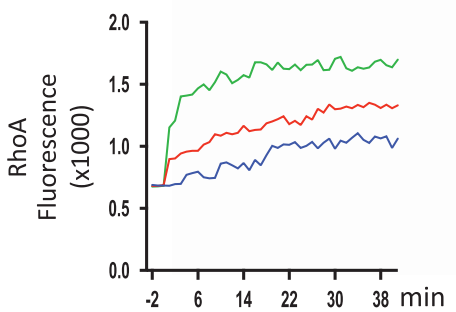

b

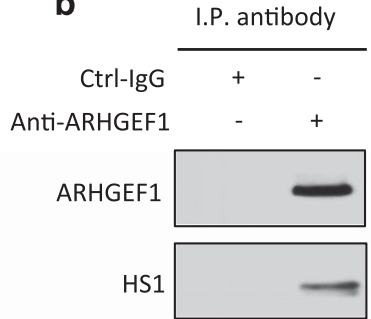

d

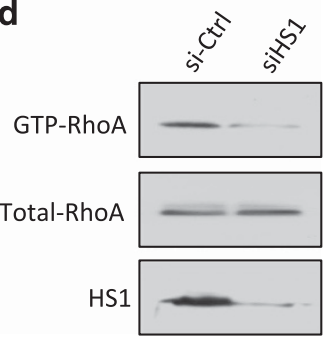

Figure 4. HS1 associates with ARHGEF1, which undergoes HS1-dependent activation to enhance activation of RhoA. (a) Immunoblot analysis of anti-HS1 ip or Ctrl-lgG ip, as indicated at the top, using lysates prepared from freshly isolated primary CLL cells; the membranes were probed with anti-HS1 or anti-ARHGEF1 antibody, as indicated on the left. (b) Immunoblot analysis of antiARHGEF1 ip or Ctrl-lgG ip, as indicated at the top, using lysates prepared from freshly isolated primary CLL cells; membranes were probed with anti-HS1 or anti-ARHGEF1 antibody, as indicated on the left. (c) In vitro exchange assay on RhoA of anti-ARHGEF1 ip from lysates of CLL cells transfected with Ctrl-siRNA (green line) or siRNA specific for HS1 (red line) in the presence of Wnt5a. The green line depicts GTPase-activation using buffer alone. (d) Immunoblot analysis of lysates prepared from MEC1-ROR1 cells transfected $72 \mathrm{~h}$ previously with control siRNA or siRNA targeting HS1; expression of HS1, total RhoA, and activated RhoA was measured, as indicated on the left.

(Supplementary Figure S5B). However, a mutant with $\mathrm{P} \rightarrow \mathrm{A}$ substitutions at 841 and 850 in the PRD domain of ROR1 was unable to associate effectively with HS1 or enhance chemotaxis of MEC1 in response to CCL21 (Supplementary Figure S5B). Thus, we generated additional mutant forms of ROR1 having a single $P \rightarrow A$ substitution at either 841 or 850 . We found that ROR1 with a $\mathrm{P}(850) \mathrm{A}$ could complex with HS1, recruit ARHGEF1, allow for HS1 phosphorylation, RhoA activation and enhanced chemokinedirected motility of MEC1 cells as effectively as W/T ROR1 (Figures $6 \mathrm{~d}-\mathrm{f}$ and Supplementary Figures S6A and B). However, ROR1 with a P(841)A substitution could not complex effectively with HS1 or ARHGEF in MEC1 cells or enhance phosphorylation of HS1 or activation of RhoA (Figures $6 \mathrm{~d}$ and e). Moreover, MEC1 cells transfected with ROR1 with a P(841)A substitution did not have enhanced chemokine-directed migration over that seen in MEC1 parental cells lacking ROR1 (Figures 6f).

\section{DISCUSSION}

Here we demonstrate that ROR1 interacts with HS1, which undergoes tyrosine phosphorylation in response to Wnt5a in primary CLL cells and in the CLL-cell line MEC1 transfected to express ROR1. We also found that ROR1/HS1 interacted with ARHGEF1, which undergoes activation, leading to the enhanced production of activated RhoA. Reducing expression of HS1 with specific siRNA inhibited Wnt5a-enhanced CXCL12-directed cells migration. As such, these studies reveal an important role for HS1 in ROR1-dependent non-canonical Wnt5a signaling important for directional migration and plantar cell polarity. a

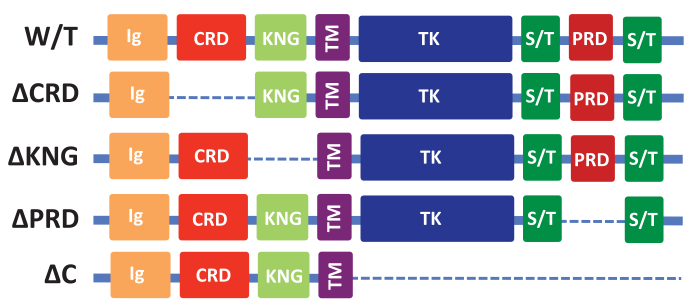

b

I.P: Anti-ROR1 antibody
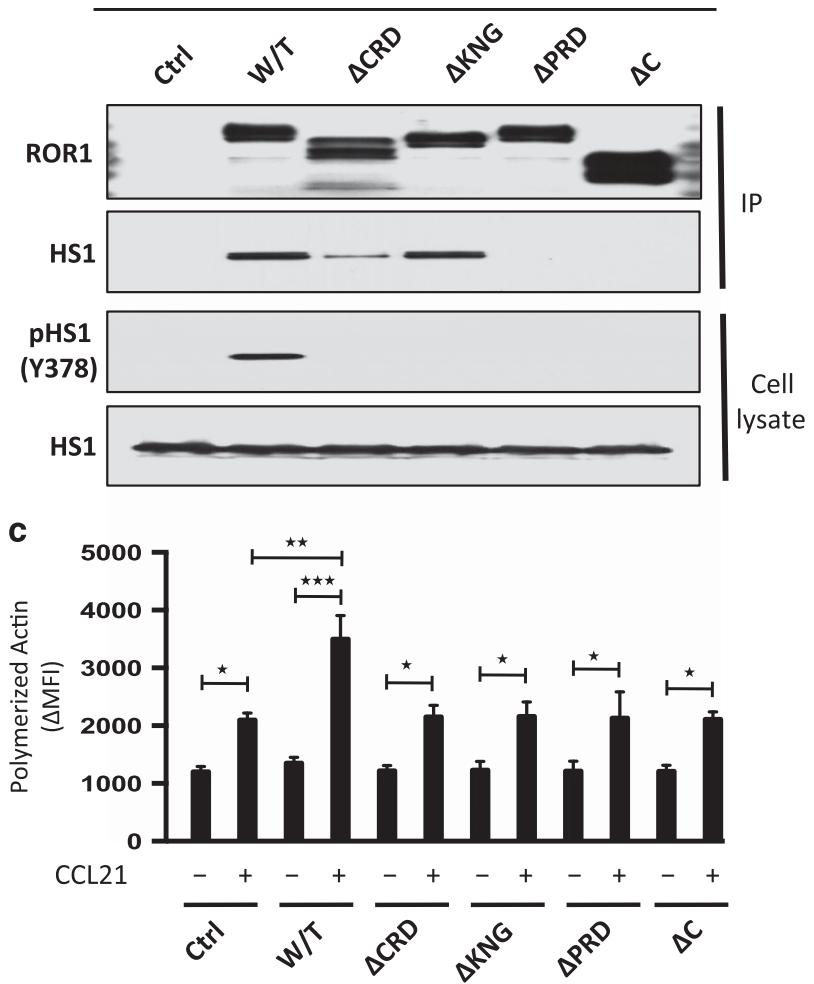

Figure 5. Structural domains of ROR1 required for it to interact with HS1 and to effect HS1 phosphorylation and enhance F-actin polymerization in response to CCL21. (a) Schematic depicts the structure of ROR1 or truncated forms of ROR1. W/T indicates wild type, PRD indicates proline-rich domain, CRD indicates cysteine-rich domain, KNG indicates Kringle domain and C indicates cytoplasmic domain of ROR1. (b) Interaction of ROR1 with HS1 was confirmed by immunoblot analysis of anti-ROR1 immune precipitates from lysates of MEC1 (Ctrl), MEC1-ROR1 (W/T) or MEC1 cells transfected with each of the various truncated forms of ROR1, as indicated on the top (upper panel). Immunoblots of the whole-cell lysates of the MEC1 (Ctrl), MEC1-ROR1 (W/T) or MEC1 cells transfected with each of the various truncated forms of ROR1, as indicated on the top, and probed with anti-HS1 or anti-phospho HS1 (Y378) antibody is provided in the lower panel. Expression and tyrosine phosphorylation of HS1 (Y378) was determined in the whole-cell lysates. (c) MEC1 (Ctrl), MEC1-ROR1 (W/T), or MEC1 cells transfected with each of the various truncated forms of ROR1, as indicated at the bottom, were examined for F-actin polymerization in the absence $(-)$ or presence $(+)$ of chemokine CCL21 $(100 \mathrm{ng} / \mathrm{ml})$. Data are shown as mean \pm s.d. from three independent experiments, $(n=5)$. ${ }^{*} P<0.05$; ${ }^{* *} P<0.01 ;{ }^{* * *} P<0.001$, as calculated using two-tailed Student's $t$-test.

Even though HS1 is a cytoplasmic protein, the extracellular domains of ROR1 contribute to the activation of HS1 in MEC1 cells. The CRD domain of ROR1 is highly conserved and serves as the putative binding site for Wnt5a, 6,32-36 whereas the KNG domain is required for ROR1 to complex with other surface proteins, such as ROR2, in response to Wnt5a. ${ }^{12}$ Deletion of either domain 
a

1
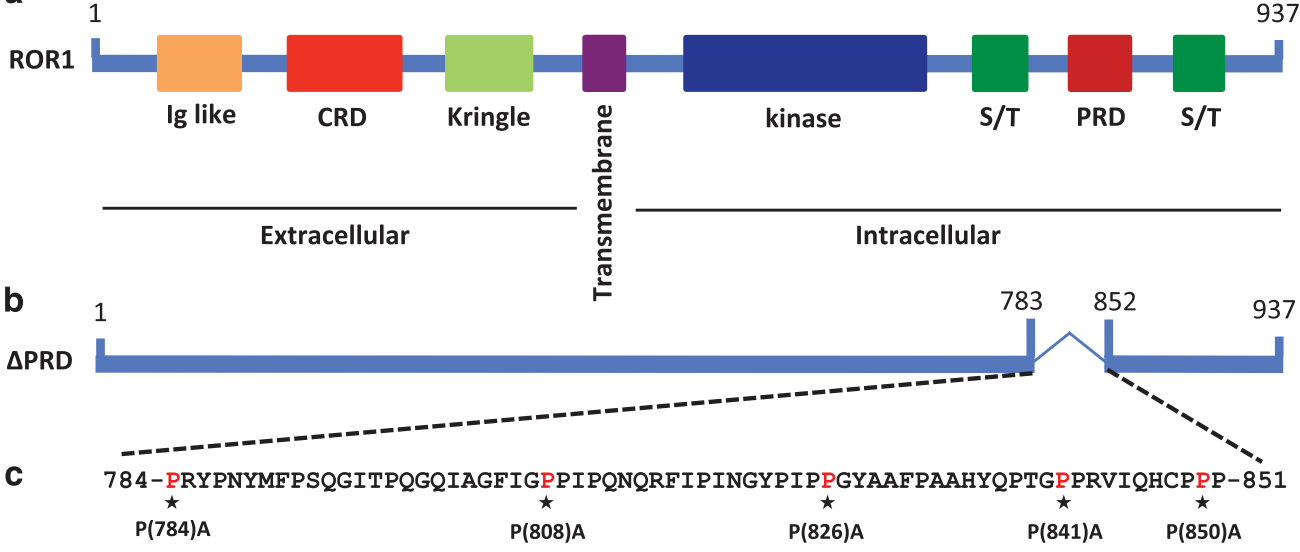

d

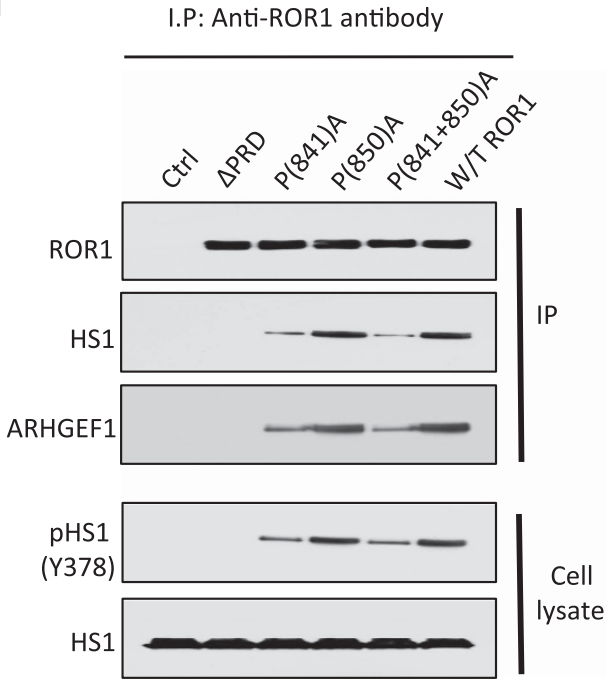

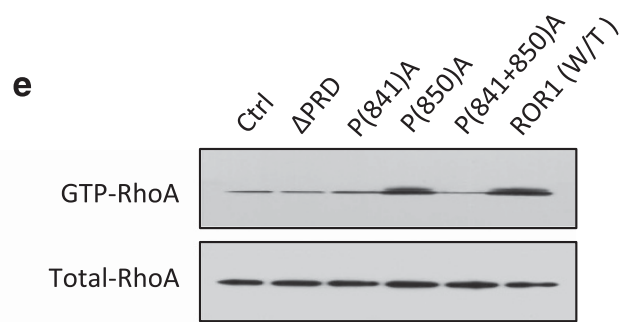

f 37 7 . 
substitutions at any other proline residue within the ROR1 PRD. As such, the proline residue at 841 appears critical for ROR1 binding to HS1, or possibly another cytosolic protein(s) that in turn associates with HS1. In any case, this residue appears necessary for ROR1 to effectively activate HS1 and enhance chemokine-directed migration of MEC1 cells.

Phosphorylation of HS1 in CLL cells isolated from the blood was associated with enhanced leukemia-cell migration and unfavorable clinical outcome. ${ }^{26,27} \mathrm{HS} 1$ can undergo phosphorylation in response to factors other than Wnt5a, such antigen or anti-lgM, which can induce B-cell receptor signaling. ${ }^{17,31}$ However, the results of our study suggests that the factor that might contribute most to HS1 phosphorylation in circulating CLL cells is Wnt5a, which is present at high levels in the plasma of patients with CLL relative to that noted in the plasma of healthy adults. ${ }^{12}$ Culture of CLL cells in media lacking Wnt5a resulted in rapid attrition of the levels of HS1 phosphorylation, which could be restored by treatment with exogenous Wnt5a. Furthermore, MEC1 cells did not have detectable phosphorylation of HS1 unless this cell line was transfected with ROR1, allowing for autocrine stimulation with Wnt5a. Finally, ibrutinib, a drug that can block BTK, could not inhibit the capacity of Wnt5a to induce phosphorylation of HS1 (Y378) or enhance the migration of CLL cells at concentrations that blocked B-cell receptor signaling. ${ }^{29,37}$ These results imply that the correlation of HS1 phosphorylation with adverse clinical outcome may reflect differences in Wnt5ainduced ROR1-dependent signaling. Relevant to this are the recent findings that high-level expression of ROR1 in CLL is associated with enhanced ROR1-signaling and shorter time from diagnosis to initial-therapy and decreased overall survival. ${ }^{10}$

Previously, we found that Wnt5a could induce recruitment and activation of ARHGEF1 to ROR1, leading to activation of RhoA. ${ }^{12}$ However, the means for how ARHGEF1 could bind ROR1 was unknown, as this GEF did not possess an SH3-binding domain. Here we provide evidence that binding of ARHGEF1 to ROR1 may be facilitated by HS1, which has been found to serve as an essential adaptor protein in T-cell-receptor signaling leading to the accumulation of $\mathrm{F}$-actin required to form the immune synapse. ${ }^{38}$ Conceivably HS1 also can serve as an adapter protein for ROR1, allowing for the recruitment and activation of ARHGEF1 and the localized activation of RhoA required for enhanced chemokine-directed cell migration. ${ }^{39}$ Consistent with this notion is the finding that Wnt5a did not enhance chemokine-directed chemotaxis of leukemia cells that had reduced expression of HS1 following treatment with specific siRNA, indicating that HS1 may play an important role in leukemia-cell migration.

For sure, HS1 does plays such a role in hemapoietic cells that lack expression of ROR1, possibly through its capacity to interact with other surface receptors, which, upon activation, require reorganization of the actin cytoskeleton for migration or cognate cell-cell interactions. ${ }^{15,16,21,31}$ Indeed, HS1 can associate with the Arp $2 / 3$ complex to facilitate F-actin polymerization. ${ }^{13,14}$ HS1-deficient T-cells have impaired cell motility, ${ }^{16}$ and fail to accumulate F-actin at the immune synapse. ${ }^{38}$ Moreover, dendritic cells from mice made deficient in $\mathrm{HS} 1\left(\mathrm{HS}^{-1-}\right)$ have aberrant lamellipodial dynamics, loosely packed podosome arrays and impaired directional migration. ${ }^{40}$ In such cases, HS1 associates with other binding partners to allow for such cellular functions following stimulation of surface receptors other than ROR1, such as CXCR4 or CCR7. ${ }^{15,16,41-44}$ In any case, our studies show that in CLL cells that express ROR1, Wnt5a can induce activation of HS1, which may cross-talk with HS1 associated with other receptors to enhance directional leukemia-cell migration. ${ }^{13,45}$

In summary, the present study describes a previously unrecognized ROR1/HS1/ARHGEF1-dependent mechanism for activating RhoA in response to Wnt5a. The findings reported here demonstrate the importance of HS1 in ROR1-dependent Wnt5a-induced signaling and highlight a pathway for potential drug development. To this end, we found that the capacity of Wnt5a to induce ROR1 to associate and activate HS1 could be blocked by cirmtuzumab, a first-in-class humanized anti-ROR1 mAb, which is being evaluated in patients with CLL (https://clinicaltrials.gov/ct2/show/NCT02222688). ${ }^{46}$ Moreover, we found that cirmtuzumab could block the capacity of Wnt5a to induce recruitment and activation of HS1 and ARHGEF1 to ROR1, contributing to the noted capacity of cirmtuzumab to block ROR1-dependent, non-canonical Wnt5a signaling responsible for enhancing leukemia-cell migration. These and other studies showing that cirmtuzumab can block survival-signaling pathways that appear unaffected by ibrutinib, ${ }^{37}$ support the rationale for clinical evaluation of this antibody in patients with CLL.

\section{CONFLICT OF INTEREST}

Cirmtuzumab was developed in the Kipps laboratory and licensed by Oncternal Therapeutics, Inc. from the University of California, San Diego.

\section{ACKNOWLEDGEMENTS}

We thank Esther Avery, Ling Zhang, Jessie-F Fecteau and Emanuela M Ghia for technical assistance. We also thank the California Institute for Regenerative Medicine (CIRM) (grant DR3-06924) for supporting us in generating anti-ROR1 mAbs and cirmtuzumab. This work was supported by the UCSD Foundation Blood Cancer Research Fund (BCRF), a SCOR grant (7005-14) from the Leukemia and Lymphoma Society and a PO1 grant (5P01CA081534-14) from the NIH for the CLL Research Consortium.

\section{AUTHOR CONTRIBUTIONS}

MKH and TJK designed research and conceived the project. MKH, JY, LC, BC, GFW and LR performed research. ZS and SPB performed mass spectrometry analysis. MKH, SPB and TJK analyzed data. MKH and TJK wrote the manuscript.

\section{REFERENCES}

1 Masiakowski P, Carroll RD. A novel family of cell surface receptors with tyrosine kinase-like domain. J Biol Chem 1992; 267: 26181-26190.

2 Wilson C, Goberdhan DC, Steller H. Dror a potential neurotrophic receptor gene, encodes a Drosophila homolog of the vertebrate Ror family of Trk-related receptor tyrosine kinases. Proc Natl Acad Sci USA 1993; 90: 7109-7113.

3 Forrester WC, Dell M, Perens E, Garriga G. A C. elegans Ror receptor tyrosine kinase regulates cell motility and asymmetric cell division. Nature 1999; 400: 881-885.

4 Rodriguez-Niedenfuhr M, Prols F, Christ B. Expression and regulation of ROR-1 during early avian limb development. Anat Embryol (Berl) 2004; 207: 495-502.

5 Broome HE, Rassenti LZ, Wang HY, Meyer LM, Kipps TJ. ROR1 is expressed on hematogones (non-neoplastic human B-lymphocyte precursors) and a minority of precursor-B acute lymphoblastic leukemia. Leuk Res 2011; 35: 1390-1394.

6 Fukuda T, Chen L, Endo T, Tang L, Lu D, Castro JE et al. Antisera induced by infusions of autologous Ad-CD154-leukemia B cells identify ROR1 as an oncofetal antigen and receptor for Wnt5a. Proc Natl Acad Sci USA 2008; 105: 3047-3052.

7 Baskar S, Kwong KY, Hofer T, Levy JM, Kennedy MG, Lee E et al. Unique cell surface expression of receptor tyrosine kinase ROR1 in human B-cell chronic lymphocytic leukemia. Clin Cancer Res 2008; 14: 396-404.

8 Daneshmanesh AH, Mikaelsson E, Jeddi-Tehrani M, Bayat AA, Ghods R, Ostadkarampour $\mathrm{M}$ et al. Ror1, a cell surface receptor tyrosine kinase is expressed in chronic lymphocytic leukemia and may serve as a putative target for therapy. Int J Cancer 2008; 123: 1190-1195.

9 Widhopf GF II, Cui B, Ghia EM, Chen L, Messer K, Shen Z et al. ROR1 can interact with TCL1 and enhance leukemogenesis in Emu-TCL1 transgenic mice. Proc Natl Acad Sci USA 2014; 111: 793-798.

10 Cui B, Ghia EM, Chen L, Rassenti LZ, DeBoever C, Widhopf GF II et al. High-level ROR1 associates with accelerated disease-progression in chronic lymphocytic leukemia. Blood 2016; 128: 2931-2940.

11 Witze ES, Litman ES, Argast GM, Moon RT, Ahn NG. Wnt5a control of cell polarity and directional movement by polarized redistribution of adhesion receptors. Science 2008; 320: 365-369.

12 Yu J, Chen L, Cui B, Widhopf GF II, Shen Z, Wu R et al. Wnt5a induces ROR1/ROR2 heterooligomerization to enhance leukemia chemotaxis and proliferation. J Clin Invest 2016; 126: 585-598. 
13 Uruno T, Zhang P, Liu J, Hao JJ, Zhan X. Haematopoietic lineage cell-specific protein 1 (HS1) promotes actin-related protein (Arp) 2/3 complex-mediated actin polymerization. Biochem J 2003; 371(Pt 2): 485-493.

14 Hao JJ, Zhu J, Zhou K, Smith N, Zhan X. The coiled-coil domain is required for HS1 to bind to F-actin and activate Arp2/3 complex. J Biol Chem 2005; 280: 37988-37994.

15 Mukherjee S, Kim J, Mooren OL, Shahan ST, Cohan M, Cooper JA. Role of cortactin homolog HS1 in transendothelial migration of natural killer cells. PLoS One 2015; 10: e0118153.

16 Lettau M, Kabelitz D, Janssen O. SDF1alpha-induced interaction of the adapter proteins Nck and HS1 facilitates actin polymerization and migration in T cells. Eur J Immunol 2015; 45: 551-561.

17 Ten Hacken E, Sivina M, Kim E, O'Brien S, Wierda WG, Ferrajoli A et al. Functional differences between $\operatorname{lgM}$ and $\operatorname{lgD}$ signaling in chronic lymphocytic leukemia. J Immunol 2016; 197: 2522-2531.

18 Kitamura D, Kaneko H, Miyagoe Y, Ariyasu T, Watanabe T. Isolation and characterization of a novel human gene expressed specifically in the cells of hematopoietic lineage. Nucleic Acids Res 1989; 17: 9367-9379.

19 Alexandropoulos K, Cheng G, Baltimore D. Proline-rich sequences that bind to Src homology 3 domains with individual specificities. Proc Natl Acad Sci USA 1995; 92 : 3110-3114.

20 Weng Z, Rickles RJ, Feng S, Richard S, Shaw AS, Schreiber SL et al. Structurefunction analysis of $\mathrm{SH} 3$ domains: $\mathrm{SH} 3$ binding specificity altered by single amino acid substitutions. Mol Cell Biol 1995; 15: 5627-5634.

21 Martinez-Quiles N. Emerging roles of hematopoietic lineage cell-specific protein 1 in the immune system. OA Immunol 2013; 1: 1-7.

22 Butrym A, Majewski M, Dzietczenia J, Kuliczkowski K, Mazur G. High expression of hematopoietic cell specific Lyn substrate-1 (HS1) predicts poor survival of B-cell chronic lymphocytic leukemia patients. Leuk Res 2012; 36: 876-880.

23 Frezzato F, Gattazzo C, Martini V, Trimarco V, Teramo A, Carraro S et al. HS1, a Lyn kinase substrate, is abnormally expressed in B-chronic lymphocytic leukemia and correlates with response to fludarabine-based regimen. PLoS One 2012; 7: e39902.

24 Muzio M, Scielzo C, Frenquelli M, Bachi A, De Palma M, Alessio M et al. HS1 complexes with cytoskeleton adapters in normal and malignant chronic lymphocytic leukemia B cells. Leukemia 2007; 21: 2067-2070.

25 ten Hacken E, Scielzo C, Bertilaccio MT, Scarfo L, Apollonio B, Barbaglio F et al. Targeting the LYN/HS1 signaling axis in chronic lymphocytic leukemia. Blood 2013; 121: 2264-2273.

26 Scielzo C, Ghia P, Conti A, Bachi A, Guida G, Geuna M et al. HS1 protein is differentially expressed in chronic lymphocytic leukemia patient subsets with good or poor prognoses. J Clin Invest 2005; 115: 1644-1650.

27 Scielzo C, Bertilaccio MT, Simonetti G, Dagklis A, ten Hacken E, Fazi C et al. HS1 has a central role in the trafficking and homing of leukemic B cells. Blood 2010; 116: 3537-3546.

28 Burger JA, Burger M, Kipps TJ. Chronic lymphocytic leukemia B cells express functional CXCR4 chemokine receptors that mediate spontaneous migration beneath bone marrow stromal cells. Blood 1999; 94: 3658-3667.

29 Di Paolo JA, Huang T, Balazs M, Barbosa J, Barck KH, Bravo BJ et al. Specific Btk inhibition suppresses B cell- and myeloid cell-mediated arthritis. Nat Chem Biol 2011; 7: 41-50.

30 Stacchini A, Aragno M, Vallario A, Alfarano A, Circosta P, Gottardi D et al. MEC1 and MEC2: two new cell lines derived from B-chronic lymphocytic leukaemia in prolymphocytoid transformation. Leuk Res 1999; 23: 127-136.

31 Yamanashi Y, Okada M, Semba T, Yamori T, Umemori H, Tsunasawa S et al. Identification of HS1 protein as a major substrate of protein-tyrosine kinase(s) upon B-cell antigen receptor-mediated signaling. Proc Natl Acad Sci USA 1993; 90: 3631-3635.

32 Roszmusz E, Patthy A, Trexler M, Patthy L. Localization of disulfide bonds in the frizzled module of Ror1 receptor tyrosine kinase. J Biol Chem 2001; 276: 18485-18490.

33 Forrester WC, Kim C, Garriga G. The Caenorhabditis elegans Ror RTK CAM-1 inhibits EGL-20/Wnt signaling in cell migration. Genetics 2004; 168: 1951-1962.

34 Masiakowski P, Yancopoulos GD. The Wnt receptor CRD domain is also found in MuSK and related orphan receptor tyrosine kinases. Curr Biol 1998; 8: R407.

$35 \mathrm{Xu}$ YK, Nusse R. The Frizzled CRD domain is conserved in diverse proteins including several receptor tyrosine kinases. Curr Biol 1998; 8: R405-R406.

36 Qi X, Okinaka Y, Nishita M, Minami Y. Essential role of Wnt5a-Ror1/Ror2 signaling in metanephric mesenchyme and ureteric bud formation. Genes Cells 2016; 21: 325-334.

37 Yu J, Chen L, Cui B, Wu C, Choi MY, Chen Y et al. Cirmtuzumab inhibits Wnt5ainduced Rac1-activation in chronic lymphocytic leukemia treated with ibrutinib. Leukemia 2017; 31: 1333-1339.

38 Gomez TS, McCarney SD, Carrizosa E, Labno CM, Comiskey EO, Nolz JC et al. HS1 functions as an essential actin-regulatory adaptor protein at the immune synapse. Immunity 2006; 24: 741-752.

39 Kurokawa K, Matsuda M. Localized RhoA activation as a requirement for the induction of membrane ruffling. Mol Biol Cell 2005; 16: 4294-4303.

40 Dehring DA, Clarke F, Ricart BG, Huang Y, Gomez TS, Williamson EK et al. Hematopoietic lineage cell-specific protein 1 functions in concert with the Wiskott-Aldrich syndrome protein to promote podosome array organization and chemotaxis in dendritic cells. J Immunol 2011; 186: 4805-4818.

41 Takemoto Y, Furuta M, Sato M, Kubo M, Hashimoto Y. Isolation and characterization of a novel HS1 SH3 domain binding protein, HS1BP3. Int Immunol 1999; 11: 1957-1964.

42 Skokowa J, Klimiankou M, Klimenkova O, Lan D, Gupta K, Hussein K et al. Interactions among HCLS1, HAX1 and LEF-1 proteins are essential for G-CSF-triggered granulopoiesis. Nat Med 2012; 18: 1550-1559.

43 Suzuki Y, Demoliere C, Kitamura D, Takeshita H, Deuschle U, Watanabe T. HAX-1 a novel intracellular protein, localized on mitochondria, directly associates with HS1, a substrate of Src family tyrosine kinases. J Immunol 1997; 158: 2736-2744.

44 Brunati AM, Donella-Deana A, James P, Quadroni M, Contri A, Marin O et al. Molecular features underlying the sequential phosphorylation of HS1 protein and its association with C-Fgr protein-tyrosine kinase. J Biol Chem 1999; 274: 7557-7564.

45 Cavnar PJ, Mogen K, Berthier E, Beebe DJ, Huttenlocher A. The actin regulatory protein HS1 interacts with Arp2/3 and mediates efficient neutrophil chemotaxis. J Biol Chem 2012; 287: 25466-25477.

46 Choi MY, Widhopf GF, Wu CC, Cui B, Lao F, Sadarangani A et al. Pre-clinical specificity and safety of UC-961, a first-in-class monoclonal antibody targeting ROR1. Clin Lymphoma Myeloma Leuk 2015; 15(Suppl): S167-S169.

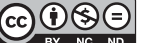

This work is licensed under a Creative Commons AttributionNonCommercial-NoDerivs 4.0 International License. The images or other third party material in this article are included in the article's Creative Commons license, unless indicated otherwise in the credit line; if the material is not included under the Creative Commons license, users will need to obtain permission from the license holder to reproduce the material. To view a copy of this license, visit http:// creativecommons.org/licenses/by-nc-nd/4.0/

(c) The Author(s) 2017

Supplementary Information accompanies this paper on the Leukemia website (http://www.nature.com/leu) 